Communautés, enjeux environnementaux et recherche en éducation relative à l'environnement

\title{
lan Robottom
}

Traducteur : Renée Brunelle, Tom Berryman et Lucie Sauvé

URL : http://journals.openedition.org/ere/4867

DOI : 10.4000 /ere 4867

ISSN : 2561-2271

Éditeur

Centr'ERE

\section{Référence électronique}

lan Robottom, «Communautés, enjeux environnementaux et recherche en éducation relative à

l'environnement », Éducation relative à l'environnement [En ligne], Volume 4 | 2003, mis en ligne le 14 septembre 2003, consulté le 16 juin 2020. URL : http://journals.openedition.org/ere/4867 ; DOI :

https://doi.org/10.4000/ere.4867 


\section{Communautés, enjeux environnementaux et recherche en éducation relative à l'environnement}

\section{Ian Robottom}

Traduction : Renée Brunelle, Tom Berryman et Lucie Sauvé

Je tiens à souligner l'appui de Geoff Fagan et de collègues de la Faculté d'éducation de l'Université de Strathclyde à Glasgow en Écosse ainsi que celui de Lucie Sauvé et de collègues de la Faculté d'éducation de l'Université du Québec à Montréal au Québec (Canada).

1 Dans cet article, je présenterai d'abord deux récents projets environnementaux centrés sur la communauté. L'un d'eux vise le développement de programmes d'éducation relative à l'environnement (ERE) alors que l'autre aborde plus directement des enjeux d'environnement et de développement. Mon principal objectif est d'explorer la nature et les caractéristiques des processus de résolution de problèmes environnementaux au sein de deux cultures différentes. Certaines implications pour la recherche en éducation relative à l'environnement seront induites de ces cas. Afin d'introduire une telle discussion, j'exposerai brièvement un point de vue sur la trajectoire récente de la recherche en ERE.

2 Le domaine de la recherche en éducation relative à l'environnement a vécu un certain nombre de changements d'orientation au cours des 25 premières années de son existence. Pendant la période des années 70 et 80 , l'approche prédominante de la recherche en éducation relative à l'environnement était nettement basée sur les sciences appliquées. La première revue internationale dans le domaine, The Journal of Environmental Education, présentait avant tout des recherches empiriques, quantitatives et de nature quasi- expérimentale et excluait presque toutes autres formes de recherche. C'était alors une période de pratiques de recherche en ERE non réflexives et peu critiques. Le domaine était alors marqué par la reproduction de pratiques de recherche bien établies (Robottom et Hart, 1995). 
3 À partir de la fin des années 80 et du début des années 90, il y eut une période de débats intenses sur la recherche en éducation relative à l'environnement (Mrazek, 1993). Les pratiques de recherche des années 70 et 80 ont été l'objet d'une réflexion critique. Les présupposés auparavant pris pour acquis ont été remis en cause et une diversité de nouvelles approches de recherche ont été identifiées et prises en compte de manière critique. Des débats méthodologiques ont été déclenchés, des arguments pour des approches alternatives ont été développés et des critiques ont été formulées.

Chevauchant cette période de débats et de métathéorisation sur la recherche, mais surtout postérieure à elle, plusieurs projets de recherche internationaux, à grande échelle et sous financement externe, ont été lancés, adoptant des approches nourries par ces débats méthodologiques. Au-delà des objectifs pour lesquels ils étaient financés, de tels projets ont également permis l'expérimentation concrète de nouvelles approches de recherche. Afin de réfléchir sur la nature, le statut et le rôle de la recherche en éducation relative à l'environnement, je vais me référer plus spécifiquement à deux projets que je choisis pour les raisons suivantes :

- Il s'agit de projets dans lesquels j'ai eu une expérience directe. Dans le premier cas, j'étais directeur du projet et dans le deuxième, j'étais responsable d'une étude de cas.

- Les projets sont récents. Le premier projet a eu lieu en 1998-1999; l'autre est en cours depuis les dix dernières années et j'y ai été impliqué en 2002.

- Les projets sont reliés au thème de ce volume. Le premier projet était un projet de développement mis en œuvre dans un contexte de changements culturels marqués et d'ajustements politiques fondamentaux. Le deuxième projet était essentiellement une étude d'efforts locaux de survie sociale et culturelle. Les deux projets traitent explicitement d'une diversité d'enjeux environnementaux.

\section{Projet AusLinks (Australia/South Africa Institutional Links)}

Le projet intitulé Educating for Socio-Ecological Change : Capacity-building in Environmental Education, focussing on South Africa's tertiary educators (« Éduquer pour le changement socioécologique : Renforcement des capacités en éducation relative à l'environnement chez les éducateurs de l'enseignement supérieur en Afrique du Sud ») était financé par AusAID (agence de coopération internationale australienne) et géré par IDP Education Australia puisque ce projet s'inscrivait dans le cadre du programme d'échanges institutionnels Australie/Afrique du Sud (Australia/South Africa Institutional Links). Ce projet se déroulait dans un contexte de reconstruction sociale et culturelle au début de la période post-apartheid de l'Afrique du Sud.

6 Les caractéristiques du projet ainsi que ses implications pour le développement professionnel en éducation relative à l'environnement ont été présentées par Lotz et Robottom (1998), LeGrange et coll. (1999) et Robottom et Kyburz-Graber (2000). Ce projet était explicitement basé sur des idées ayant émergé de la période de débats méthodologiques mentionnée plus haut. Plutôt que de suivre un seul processus prédéfini de développement professionnel ou d'adopter et d'adapter un seul ensemble de matériel éducatif préexistant en éducation relative à l'environnement, l'approche 
retenue fut simplement de travailler avec souplesse en fonction d'un ensemble de principes relatifs au développement professionnel. Ce dernier devrait être :

- contextuel : le développement professionnel est intimement relié aux milieux de travail et aux enjeux qui concernent les participants ;

- répondant : les enjeux traités dans les processus de développement professionnel sont ceux qui intéressent et préoccupent les participants;

- émergeant : les savoirs professionnels qui ont le plus de poids dans les discussions relatives aux façons d'améliorer la pratique professionnelle sont ceux qui émergent du travail d'étude de cas et qui se situent au cœur du processus d'autodéveloppement professionnel ;

- participatif : les participants sont directement impliqués et de la manière la plus équitable possible, dans toutes les dimensions du processus de développement professionnel (par exemple : l'identification des enjeux à aborder ; la collecte et l'analyse des données d'étude de cas ; le développement et la diffusion du matériel et des rapports);

- critique : les processus de développement professionnel vont au-delà des activités pour en questionner les dimensions politique, organisationnelle et pratique afin d'identifier et d'analyser les valeurs, les présupposés et les intérêts qui caractérisent et justifient ces activités ;

- praxéologique : les processus de développement professionnel se déroulent au travers de la praxis et sont médiatisés par celle-ci. Au sein de ce projet, la praxis est définie comme une interaction réflexive entre la théorie professionnelle-personnelle, la pratique professionnelle-personnelle et les contextes professionnels où ces dernières théories et pratiques deviennent intelligibles (Robottom et Kyburz-Graber, 2000).

7 Le projet AusLinks mettait l'accent sur le développement professionnel des acteurs de l'éducation relative à l'environnement dans les institutions d'enseignement supérieur participantes. Le projet cherchait à améliorer les capacités professionnelles en matière d'intervention éducative et de recherche, en travaillant entre collègues dans un processus de recherche participative axé sur le milieu de travail et visant le développement d'études de cas documentant des changements de pratiques en matière d'éducation relative à l'environnement.

Dans le projet AusLinks, le développement de telles études de cas fut conceptualisé en tant que pivot central pour le développement professionnel de deux manières distinctes. Premièrement, le processus de développement des études de cas fut conçu comme une stratégie d'autodéveloppement professionnel alors que les participants mènent une réflexion critique sur la signification et le sens de leurs théories, des conditions organisationnelles, des politiques et de leurs pratiques d'enseignement. Deuxièmement, les études de cas furent conçues en tant que matériel de base ou point de départ pour de futures interactions de développement professionnel avec d'autres éducateurs (non-participants au projet).

9 Le processus mis en œuvre dans le projet est décrit dans la présentation suivante, faite par certains des participants lors de la conférence de leur association professionnelle nationale en 1999 :

En petits groupes, les participants ont d'abord échangé sur un certain nombre d'enjeux environnementaux et d'éducation relative à l'environnement. Il a été décidé par consensus que l'activité consisterait à développer des études de cas reliées aux contextes professionnels des participants et situées dans le contexte géographique de leurs lieux de travail respectifs. Des lignes directrices ont été suggérées par les animateurs de l'activité afin de fournir une structure initiale de base et certaines idées de départ. Ce processus devait inclure le développement de 
banques de photos de personnes, de lieux, de contextes et d'activités à partager lors de la prochaine réunion. À cette fin, des appareils-photo ont été fournis aux participants.

Lors de la deuxième réunion tenue dans la Province du Nord, chaque participant a soumis à la discussion des photos illustrant certains aspects des réalités qu'il estimait importants parce que porteurs de signification et de sens au regard du cas étudié. D'autres participants à cette " activité 2 » ont réagi à ces rapports illustrés de photos, permettant l'identification d' écarts" ou de lacunes dans les façons d'illustrer chaque étude de cas.

Ces banques de photos se sont avérées importantes pour faciliter la poursuite des discussions visant à élaborer encore davantage les études de cas. Lors de la troisième réunion, les photos ont été dotées de légendes par les différents participants et celles-ci ont été partagées et discutées avec les autres participants afin de poursuivre le développement et l'amélioration du texte. Lors de cette réunion, les participants ont également commencé à élaborer des commentaires sur les études de cas qui seraient présentés lors de la quatrième réunion.

Lors de cette dernière, les ébauches d'études de cas comprenaient des commentaires (5 à 10 pages) et des photos accompagnées de légendes (5 à 10 lignes). Ces ébauches d'études de cas furent partagées avec au moins deux autres participants qui ont formulé verbalement et par écrit des commentaires critiques sous forme d'annotations sur le texte. Des réactions ont également été formulées lors d'une session plénière : tous les participants ont été impliqués dans une discussion au sujet du développement de la trame narrative des études de cas. Soulignons qu'à ce stade du processus, les relations de confiance étaient assez bien établies entre les participants; ce niveau de confiance a facilité le processus de collaboration critique qui était au centre de notre démarche d'écriture des études de cas.

Toutes les institutions ont été visitées durant le projet. La réunion de planification a eu lieu à l'Université de Stellenbosch, la deuxième réunion dans la Province du Nord, la troisième à Grahamstown et la quatrième dans la Province du Nord-Ouest. Les lieux de travail de tous les participants ont ainsi pu être visités (Université de Venda, Collège d'éducation Tshisimane, Collège d'éducation Shingwedzi et Collège d'éducation Tlhabane). Nous avons également pu visiter certains des sites sous enquête par un certain nombre de participants, surtout dans la Province du Nord. (LeGrange et coll., 1999, p. 3-5, traduction libre)

10 Les institutions participantes ont toutes développé des études de cas portant sur le réseautage des enseignants en éducation relative à l'environnement ainsi que sur le développement curriculaire axé sur des enjeux environnementaux pour les études avancées. Les cas étudiés sont caractérisés par leur diversité et par leur sensibilité au contexte.

11 Dans la Province du Nord, l'étude de cas sur le réseautage des enseignants met en relief les forces et les faiblesses d'un réseau d'enseignants et fournit des éléments de réflexion utiles pour l'établissement et l'appui de réseaux d'enseignants en éducation relative à l'environnement. L'étude de cas sur le développement d'un curriculum axé sur des enjeux environnementaux au Collège shingwedzi présente différentes dimensions des enjeux concernés. Ce cas permet d'entrevoir comment un curriculum peut être développé autour de tels enjeux. Dans la Province du Nord-Ouest, les enseignants du Collège Tlhabane ont développé une étude de cas portant sur l'épidémie de SIDA/VIH dans la région de Rustenberg en tant que point focal pour un développement curriculaire des études avancées. Ce cas souligne l'importance de considérer le contexte local dans un tel curriculum. Dans la région du Cap-Ouest, les enseignants de l'Université Stellenbosch ont dirigé deux programmes de formation des enseignants. Ces programmes mettent en lumière la nécessité de prendre en compte les 
enjeux de relations et de pouvoir dans les processus pédagogiques constructivistes en éducation relative à l'environnement, centrés sur les sciences et la viabilité. Dans la région du Cap-Est, les enseignants de l'Université Rhodes ont développé un cours en éducation relative à l'environnement destiné aux étudiants des études supérieures en éducation. Ce cas illustre les tensions entre les processus d'éducation relative à l'environnement et les contraintes contextuelles des institutions d'études avancées. Il constitue également un exemple d'une approche thématique pour le développement curriculaire au niveau de l'enseignement supérieur (LeGrange et coll., 1999).

Plusieurs retombées utiles ont émané de ce projet de recherche participative, incluant notamment : une plus grande confiance et des capacités accrues chez plusieurs participants ; une plus grande disponibilité, en Afrique du Sud, de matériel et de ressources en provenance du contexte australien ; le développement de nouveau matériel contextuellement pertinent pour les Sud-Africains ; la mise en place, l'extension et l'approfondissement de réseaux de collégialité ; en fin de compte, une meilleure appréciation de ce qui peut être accompli grâce au travail collaboratif impliquant des représentants d'une diversité d'institutions d'enseignement supérieur en Afrique du Sud. Des liens ont été établis entre les participants sud-africains, ainsi qu'avec les partenaires australiens, dans un domaine où de tels liens n'existaient pas encore. Autre fait important à souligner, depuis la fin de ce projet, certains participants sud-africains occupent dorénavant des postes de responsabilité dans les domaines du développement curriculaire et du développement professionnel en éducation relative à l'environnement en Afrique du Sud (Reddy, 2002).

13 La portée de ces retombées positives peut être mieux comprise lorsqu'on les considère au regard de l'histoire récente de la vie sociale sud-africaine telle qu'elle se dessinait avant le début de ce projet. Au cours des décennies qui ont précédé les années 90 , un gouvernement minoritaire blanc, via les politiques ségrégationnistes de l'Apartheid, a systématiquement opprimé et nié les possibilités éducatives des personnes de couleur. L'éducation elle-même était un instrument pour développer et maintenir un ensemble de structures et de relations de pouvoir dans lesquelles les personnes de couleur étaient sérieusement désavantagées. Le projet AusLinks a été conçu afin d'aborder et possiblement de contribuer à corriger, dans la mesure du possible, certaines de ces injustices historiques, et ce, dans le domaine particulier de l'éducation relative à l'environnement. C'est pour cette raison que les approches participatives et critiques de la recherche en éducation ont été adoptées en tant que fondements théoriques de ce projet de développement professionnel. Nous estimions qu'une approche de recherche caractérisée par des principes de sensibilité au contexte, de réceptivité et de praxis critique était nécessaire afin de mettre en place les conditions pour que des personnes précédemment désavantagées génèrent leur propre vision de l'éducation et construisent leur propre curriculum en cohérence avec cette vision (LeGrange et coll., 1999).

\section{Le projet CADISPA en Écosse : Coopération et développement en régions peu peuplées}

Le programme CADISPA (Cooperation and Development in Sparsely Populated Areas) est coordonné par Geoff Fagan au sein du Département d'éducation communautaire de la Faculté d'éducation de l'Université de Strathclyde à Glasgow, en Écosse. 


\section{Buts et principes de CADISPA}

Selon un document récent présentant le projet CADISPA (décembre 2001), celui-ci vise à développer une définition de la viabilité (sustainability) qui sera utile aux populations locales et à la communauté économique. L'accent est nettement mis sur la participation et sur le caractère local, comme en témoignent les extraits suivants :

Pour CADISPA, toute régénération économique et sociale doit émerger d'un cadre de référence qui prend en compte les facteurs de viabilité économique, environnementale et sociale. CADISPA bâtit le capital social grâce à l'éducation non formelle, l'implication active et la prise de décision locale. CADISPA utilise les stratégies courantes de développement communautaire afin d'aider les personnes au sein de communautés spécifiques à s'approprier, comprendre et agir en fonction de leur propre programme de viabilité. (CADISPA, 2001, p. 1, traduction libre)

Le but du modèle CADISPA est d'aider les communautés locales à identifier leurs besoins de développement et à les soutenir alors qu'elles cherchent à mettre en place leur programme collectif et à développer des partenariats avec les agences de développement économique et avec les autorités locales. (Hampson et Fagan, 1997, p. 1, traduction libre)

CADISPA bâtit des partenariats ruraux en cherchant à identifier un point d'entrée reconnu par les communautés locales et en travaillant toujours avec les groupes communautaires existants. CADISPA construit avec eux un programme de développement pour leur localité. CADISPA accompagne les populations locales dans la clarification de leur vision du futur - précisant graduellement l'image de ce futur et élargissant les bases de la consultation et du partenariat jusqu'à ce que chacun se sente confiant à la fois vis-à-vis du développement potentiel ainsi que vis-à-vis de sa pertinence, de son envergure, de son coût et de son caractère culturel. (Hampson et Fagan, 1997, p. 1, traduction libre)

Ce projet est axé sur la participation et le partage du pouvoir : les gens sont au centre de l'identification de leur programme d'action et de ses priorités. Ce sont eux qui déterminent les priorités et décident de leur propre développement local. Ainsi, CADISPA est associé aux discours de l'éducation relative à l'environnement portant sur la communauté, l'environnement et les approches participatives vis-à-vis du développement ; il peut potentiellement contribuer à enrichir ce discours et y porter un regard critique. De ce fait, le projet CADISPA est étroitement lié au thème spécifique de ce volume : «Environnements, cultures et développements ».

Les membres de CADISPA ont adopté un rôle d'animation. Ce rôle réside dans l'identification d'occasions de mise en projet, l'établissement de liens entre des groupes communautaires et des agences de soutien et de financement de divers types, l'établissement de statuts et règlements afin d'assurer une protection légale aux groupes communautaires et la mise en place d'un esprit de travail positif et stimulant au sein de projets complexes et souvent problématiques. Les animateurs de CADISPA assistent à plusieurs réunions de planification des groupes communautaires, bien que leur rôle soit généralement discret et réservé plutôt que très visible et directif. Mettant à profit le renforcement des capacités offert par CADISPA, les groupes communautaires sont incités à se manifester par un "acte de présence " qui soit viable et durable. Cet acte est souvent signé dans la brique et le mortier sous la forme d'un édifice communautaire ayant une valeur immédiatement fonctionnelle pour la communauté ainsi qu'une valeur iconique persistante. 

était autrefois le centre de l'industrie minière écossaise d'ardoise. La population permanente de lîle d'Easdale est de seulement 27 personnes et il n'y a aucune voiture sur l'île, à laquelle on accède par un petit bateau passeur à pont ouvert.

21 Un des développements en cours sur l'île d'Easdale est la rénovation et l'agrandissement de la salle de rencontre autrefois utilisée par l'industrie minière. Cette salle a été achetée par un résident en 1996 et remise à la Fondation de l'île d'Easdale, Eilean Eisdeal. Cette fondation est une organisation de charité dont le but explicite est celui de rénover la salle pour l'usage des habitants de l'île, afin de renforcer le tissu social, économique et environnemental de la communauté. La Fondation a réussi à obtenir une subvention importante (£700 000) pour effectuer des rénovations et aménagements et pour embaucher un-une gestionnaire pendant deux ans. Un plan architectural et un plan d'affaires ont été développés et les travaux de construction ont débuté. La vision commune d'une salle communautaire rénovée est maintenant en voie de se concrétiser. En ce sens au moins, le cas de l'île d'Easdale raconte l'histoire d'une réussite.

Les animateurs de CADISPA travaillent également avec les communautés au développement de leur propre étude de cas, décrivant l'histoire naturelle et sociale de environnementaux. Dans un processus itératif, les rapports documentant ces études de cas sont retournés aux comités communautaires pour rétroactions.

description qui en est faite met en relief les activités des communautés en projet dans deux des Îles Argyll de la côte ouest de l'Écosse et souligne certaines caractéristiques des enjeux environnementaux et communautaires en question.

\section{L'île d'Easdale}

L'Île d'Easdale est également une histoire de réussite pour d'autres motifs. L'un d'eux a trait au fait qu'on y a maintenant une meilleure compréhension des processus de développement communautaire (par exemple, le pouvoir provenant de l'action collective et la valeur de la mise en réseau avec des agences de soutien). Un autre signe de réussite a trait à une meilleure compréhension de la nature à double tranchant des projets de viabilité («sustainability») : pour que la salle communautaire génère suffisamment de revenus afin d'assurer sa viabilité économique à moyen et à long terme, elle devra également être disponible pour location à des fins commerciales auprès de groupes non- insulaires. Un enjeu a trait au fait que si la salle communautaire réussit à générer les revenus nécessaires en attirant des visiteurs externes, il peut ainsi $\mathrm{y}$ avoir une menace potentielle sur les qualités mêmes ayant attiré les résidents au départ (isolement, population clairsemée, paix et tranquillité). Bref, les actions visant à assurer la stabilité économique ont également le potentiel de menacer la stabilité environnementale et sociale. La recherche d'un équilibre entre ces intérêts divergents pourrait être le prochain défi auquel fera face la communauté de l'île d'Easdale. 


\section{L'île Tiree}

23 La population de l'île Tiree, actuellement d'environ 700 personnes, est à la baisse. On y dénombre deux fois plus de personnes âgées que la moyenne nationale, alors qu'il y a deux fois moins de personnes entre 18 et 50 ans comparativement à la moyenne nationale. On estime qu'il n'y a pas assez d'emplois professionnels sur l'île pour y retenir les jeunes diplômés. Les jeunes qui restent sur l'île tendent à le faire afin de demeurer à la ferme familiale ou encore ce sont ceux qui abandonnent les études assez tôt. Les gens qui souhaitent poursuivre une carrière professionnelle quittent l'île pour l'université ou toute autre institution de formation et ils tendent à ne pas y retourner une fois leurs études complétées. La structure d'âges de la population de l'île est un facteur important qui influence le développement des projets d'environnement et de développement.

En vue, entre autres, d'établir des infrastructures additionnelles sur l'île afin d'augmenter le potentiel d'activité commerciale et, par conséquent, le nombre d'emplois pour les insulaires, le comité communautaire a décidé de chercher du financement pour la reconstruction et l'agrandissement de sa salle communautaire. Tiree a une salle communautaire depuis la construction d'une base militaire sur l'île pendant la Deuxième Guerre mondiale. Le bâtiment servait à l'origine de cantine. Avant la guerre, les insulaires tenaient généralement leurs réunions dans les églises. Cependant, elles ne constituaient pas toujours des lieux de rencontres appropriés. Ainsi, lorsque la guerre prit fin, la cantine fut convertie en hôtel de ville. C'était le lieu pour des danses et autres activités similaires. Au fil des ans, le bâtiment a été l'objet de rénovations mineures, par exemple on y a installé un plancher plus approprié pour les danses. Son comité de gestion le louait pour des fêtes et des mariages, générant ainsi un revenu croissant d'année en année.

Cependant, le bâtiment n'avait pas été conçu pour avoir une longue durée de vie et son état s'est détérioré avec le temps. La communauté se dota d'un comité de salle communautaire qui commença à chercher du financement pour la restauration du vieux bâtiment. Le processus fut très problématique dû à des changements de composition des membres au sein du comité, à la nécessité de changements de plans (et d'architectes) et à des difficultés de communications entre le comité, l'architecte, les fournisseurs et les agences de financement.

Néanmoins, une subvention de $£ 500000$ a finalement été obtenue. La démolition du vieux bâtiment a débuté et des tranchées ont été creusées pour les nouvelles fondations. L'optimisme était élevé.

Puis la grande aciérie (dont on prévoyait qu'elle fournirait gratuitement des matériaux d'une valeur de $£ 40000$ ) fit faillite. On n'accorda au groupe communautaire qu'un seul mois pour réviser son plan en fonction de la non- disponibilité des matériaux qui devaient être fournis gratuitement par l'aciérie. Toutefois, parce que l'architecte travaillait alors sur un grand projet international outre-mer, le comité n'a pu se conformer à cette exigence. La grosse subvention que la communauté avait obtenue fut alors perdue et les fonds restants furent redistribués dans d'autres projets. Le groupe communautaire s'est dissout. Le vieux bâtiment n'existe plus maintenant et le lieu principal de réunion pour des danses et autres activités sociales a donc lui aussi disparu. Il reste maintenant un sentiment profond de déception et un tas de débris de démolition. 
28 Malgré cette situation difficile, la communauté a tout de même mis sur pieds un nouveau groupe afin de poursuivre la recherche de financement pour reconstruire la salle communautaire. Par son expérience antérieure, la communauté a développé une vision plus juste de ce que signifie être membre d'une communauté, de ce que signifie travailler ensemble pour atteindre un but commun, pour mobiliser des ressources disponibles afin de réaliser un tel but. La communauté est plus consciente également des pièges qui peuvent survenir. Il demeure un sentiment de confiance en la capacité de la communauté à générer de l'argent localement via des activités endogènes. Il y a un espoir à l'effet que la nouvelle salle, une fois construite, générera des revenus adéquats pour la tenue d'activités sociales. Le cas de L'île Tiree pourrait encore devenir l'histoire d'une réussite...

\section{Enjeux}

De diverses manières, nous sommes tous en lien avec l'environnement, quelle que soit la représentation que nous construisions de ce dernier. Nous sommes tous affectés lorsque notre environnement change. Face à des propositions de modifications de l'environnement, il y a presque toujours des divergences d'opinions à propos du si et du comment on devrait changer l'environnement avec lequel nous sommes en relation. Lorsqu'il y a des divergences d'opinions au sujet des propositions de modification ou de conservation de l'environnement, nous sommes en présence d'un «enjeu environnemental» (environmental issue) particulier. L'exploration de tels enjeux environnementaux constitue un axe important d'une éducation visant une meilleure compréhension de nos rapports avec l'environnement (Alblas et coll., 1994; House et coll., 1994 ; Izadi et Kurtakko, 1994 ; Boschhuizen et Brinkman, 1995 ; Aleixandre et Gayoso, 1996 ; Dove, 1996 ; Harris et Robottom, 1997). Les projets d'environnement et de développement décrits précédemment illustrent clairement que les enjeux environnementaux partagent certaines caractéristiques communes. Afin de faire des choix éclairés et négociés en ce qui a trait au développement curriculaire en éducation relative à l'environnement, au développement professionnel ainsi qu'à la recherche, je soutiens que nous devons adopter une approche réflexive au sujet de la nature de ces enjeux environnementaux - en les abordant comme des points de départ de délibérations curriculaires en éducation relative à l'environnement (Robottom, 1996).

Les projets Auslinks et CADISPA montrent que les enjeux environnementaux sont complexes et contextuels, controversés, ainsi que politiquement et socialement construits. Ceci ne signifie pas que de tels enjeux doivent être évités en éducation relative à l'environnement. Cependant, cela peut signifier qu'un curriculum qui ne viserait qu'à transmettre et à faire acquérir des connaissances et des compétences instrumentales pourrait s'avérer inadéquat face à la prise en compte de telles questions. Les enjeux environnementaux ne sont pas des objets à transmettre ; ils sont des constructions personnelles et sociales intelligibles à l'intérieur de contextes complexes. Ces contextes sont complexes parce qu'ils partagent généralement les caractéristiques suivantes :

- plusieurs niveaux de gouvernance sont impliqués ; chacun cherche à contrôler la nature de la résolution de la problématique (par exemple, dans l'un des sites de projets CADISPA où la communauté tente de restaurer sa salle communautaire, il y a quatre entités de gouvernance impliquées : la fondation de la salle qui en détient le bail, le comité de salle communautaire qui a la responsabilité des opérations quotidiennes de la salle, la compagnie 
villageoise créée pour gérer les transactions financières du comité et enfin, le conseil communautaire) ;

- une diversité d'acteurs clés de la communauté sont impliqués, ayant des visions spécifiques et des intérêts particuliers face à l'enjeu en question; seul un accord partiel existe au sujet d'une «vision commune" pour la résolution de la problématique (en plus des groupes formellement impliqués dans la gouvernance, il y a différents résidents, des touristes, des agences gouvernementales locales et régionales ainsi que des agences de financement qui ont un certain degré d'intérêt par rapport aux résultats) ;

- la résolution de la problématique fait appel à de sérieuses négociations entre des intérêts environnementaux, sociaux, culturels, commerciaux et politiques (dans le cas de certains projets CADISPA, de telles négociations peuvent s'étendre sur plusieurs années) ;

- il y a des problèmes de communications au sein des groupes impliqués dans la planification ainsi qu'entre ces derniers et les agences gouvernementales et, ou de financement (au sein de la communauté insulaire de l'un des projets CADISPA, la communauté établie dans le nord de l'île est géographiquement isolée de la communauté établie dans le sud de l'île et seul un sentier difficile à emprunter existe pour les relier, rendant ainsi les rencontres plus difficiles) ;

- l'expertise locale est limitée en ce qui a trait aux capacités professionnelles des personnes désireuses de s'impliquer (certaines communautés ont de très petites populations, d'autres ont des populations plus nombreuses, mais avec une faible diversité d'expertises ; dans toutes les communautés, le processus d'implication dans les projets constitue en soi une expérience de renforcement des capacités) ;

- on observe différentes compréhensions des enjeux environnementaux, elles-mêmes partiellement forgées par l'histoire éducationnelle, politique et culturelle du lieu et de la région.

31 Les enjeux environnementaux sont en effet associés à des controverses. Tout enjeu (environnemental ou autre) est caractérisé par la divergence des opinions des protagonistes. Selon le dictionnaire australien The Macquarie Dictionary de 1981, un enjeu (issue) est « un élément questionné ou contentieux, par exemple entre les parties impliquées dans une action en justice » et « un élément ou un objet au sujet duquel la prise de décision est d'une importance particulière ou publique » (traduction libre). Dans ces définitions, les « parties impliquées » dans le contentieux et qui lui accordent une « importance particulière ou publique » sont des groupes humains. Par conséquent, un événement environnemental (ou une proposition concernant un tel événement) devient un enjeu seulement lorsqu'il y a controverse et lorsque sa résolution est jugée importante par les êtres humains : on y trouve une divergence de points de vue exprimés par des gens ayant des intérêts différents, reflétant des valeurs différentes (Robottom, 1987). En ce sens, un enjeu environnemental est une construction humaine/ sociale qui a un sens dans un contexte social, culturel et politique particulier. Les enjeux environnementaux n'existent pas à l'extérieur de la conscience humaine et ne possèdent pas une existence ontologique indépendante (Robottom, 2000).

Les enjeux environnementaux qui caractérisent les projets décrits précédemment présentent une forme de tension entre les intérêts économiques et environnementaux. Dans les projets CADISPA, cette tension s'est exprimée de la façon suivante : dans la mesure où les projets réussissent à générer les revenus nécessaires pour permettre une stabilité économique, il devient dès lors possible que les qualités mêmes ayant attiré les résidents à s'installer dans ces lieux (isolement, faible densité de population, paix et 
tranquillité) soient menacées. Les projets montrent que la stabilité économique doit être réconciliée de façon concrète avec la stabilité environnementale et on doit s'attendre à ce qu'il s'agisse là d'un processus difficile. De plus, l'expérience de chacun de ces deux projets montre qu'un engagement dans les enjeux environnementaux doit inclure la prise en compte d'une diversité de réalités sociales, historiques, culturelles et politiques ; il importe également d'adopter les valeurs d'équité et de démocratie participative, et de respecter les droits humains générationnels et intergénérationnels (par exemple, sur un site CADISPA, le projet fut basé sur une vision de la situation souhaitée pour la communauté dans un horizon de 20 ans, soit plus ou moins une génération).

Comment une telle perception des enjeux environnementaux - basée sur l'expérience vécue au sein des projets décrits précédemment - est-elle liée aux écrits portant sur l'environnement et le développement?

Il convient tout d'abord d'établir que ces discussions, ces débats, ces divergences et ces contestations, bien que parfois difficiles à vivre, ne sont pas indésirables ou d'une certaine manière, pathologiques. Ils sont les moyens mêmes par lesquels se négocie une compréhension plus complexe et plus juste des problématiques environnementales au sein de chaque communauté. Barkin (2000) reconnaît le caractère ardu de ces négociations alors qu'il traite des enjeux liés à la viabilité :

La viabilité n'a pas « simplement » trait à l'environnement, à la justice économique et au développement. Elle concerne également les êtres humains et notre survie, celle des individus et celles des cultures. La viabilité [...] a trait à la lutte pour la diversité dans toutes ses dimensions. (Barkin, 2000, traduction libre)

L'expérience de CADISPA montre que lorsque les communautés cherchent activement à développer une vision partagée ou commune du développement de leur propre projet, on peut s'attendre à les voir manifester, selon Diduck (1999), quatre caractéristiques de la gestion des ressources et de l'environnement : le changement, la complexité, l'incertitude et le conflit. Cet auteur poursuit en précisant que "tout comme des personnes bien honnêtes pourraient raisonnablement être en désaccord au sujet des formes actuelles d'utilisation de ressources, elles pourraient également être en désaccord en ce qui concerne une vision appropriée du futur» (Diduck, 1999, traduction libre). Les projets CADISPA suggèrent que l'existence de tels désaccords persistants pourrait être une caractéristique des projets de développement durable (sustainability projects) - peut-être en raison de la nature contradictoire du concept luimême. Par exemple, à la lumière de l'expérience de l'̂̂le d'Easdale, il apparaît comme un non-sens de traiter de la "soutenabilité » ou du « développement durable » comme s'ils représentaient une situation future unifiée et logique pouvant être éventuellement atteinte : précisément parce que la viabilité (ou le développement durable) implique des intérêts divergents, sinon des intérêts mutuellement exclusifs (intérêts environnementaux, intérêts sociaux et intérêts économiques), un "développement durable " correspondant à la satisfaction équitable de chacun de ces intérêts est en réalité impossible. Ultimement, quelque chose doit céder. Dans le cas de l'île d'Easdale, un développement économique sous la forme d'une hausse des revenus de tourisme pourrait très bien mener à une dégradation du caractère environnemental de ce lieu, lié à son isolement, affectant de ce fait sa culture. Ainsi, les difficultés concrètes et les problématiques de terrain au sein des projets d'environnement et de développement s'expliquent par le sens même de l'entreprise : une réconciliation bien concrète de deux intérêts le plus souvent opposés (économiques et environnementaux). 

d'environnement et de développement, souligne l'importance de la participation et du contrôle local dans la prise de décision. Il fait également une observation importante, particulièrement évidente dans le projet AusLinks, mais également dans certains sites CADISPA, au sujet de la nécessité d'adopter une perspective politisée - dans laquelle le rôle de l'« élite", relativement plus puissante, est bien compris et remis en cause alors que les groupes locaux déterminent leur propre futur. Barkin (2000) met également l'accent sur le besoin de réorganiser les structures et les rapports de pouvoirs politiques et économiques dans les contextes où les gens cherchent à résoudre leurs problématiques sociales, économiques et environnementales. L'auteur poursuit en signalant que les élites doivent prendre conscience de la nécessité de favoriser la participation locale en intégrant les gens concernés dans de véritables structures de pouvoirs, de façon à confronter les principaux problèmes de l'heure ; ceci requiert une redistribution du pouvoir politique et économique, un préalable fondamental à n'importe quel programme de développement.

Les projets AusLinks et CADISPA renforcent la perspective de Barkin (2000) selon laquelle nous devons aborder l'écodéveloppement (environmental development) dans une perspective de complexité, selon une approche politique (au sens de reconnaître le besoin de changements dans les structures et les rapports de pouvoirs) et en reconnaissant le nécessaire engagement d'un réseau d'acteurs locaux dans un processus continu de réconciliation d'intérêts économiques, sociaux et environnementaux, qui correspond finalement à un combat pour la survie culturelle.

Ainsi, en résumé, l'analyse de ces deux projets, appuyée par des écrits pertinents, suggère que les enjeux environnementaux (environmental issues) présentent les caractéristiques suivantes :

- leur structure est complexe ;

- ils ont un caractère contextuel ;

- ils impliquent une vaste gamme d'acteurs clés ;

- ces acteurs clés expriment une grande diversité de valeurs et d'intérêts ;

- leur résolution fait appel à une approche politique ;

- leur résolution requiert la négociation et la réconciliation, qui sont habituellement des processus difficiles;

- le processus de résolution est fonction d'éléments sociaux, culturels, politiques et environnementaux et il s'agit souvent de cas de « survie culturelle»;

- par-dessus tout, les enjeux environnementaux sont socialement construits et doivent être reconnus et abordés ainsi.

Pour une éducation relative à l'environnement axée sur le traitement d'enjeux environnementaux, il importe de reconnaître que ces derniers n'existent pas en tant que faits à transmettre, mais sont plutôt des constructions humaines à explorer. En faisant observer que les propositions de changements environnementaux servent certains intérêts sociaux et économiques particuliers qui doivent être évalués de façon critique, Fien (1997) affirme que «les environnementalistes et les éducateurs en environnement qui cherchent des voies pour soutenir les transformations sociales ont un rôle important à jouer dans la critique de ces intérêts, afin de dégager un certain espace permettant à nos étudiants et nos communautés de découvrir leurs propres voies » (traduction libre). 


\section{Implications pour la recherche en éducation relative à l'environnement}

Quelles sont les implications de la prise en compte des caractéristiques des enjeux environnementaux pour la recherche en éducation relative à l'environnement? Quelles formes de recherche sont épistémologiquement, ontologiquement, politiquement et pratiquement cohérentes avec une telle perception des enjeux environnementaux ? Il s'agit là de questions difficiles à traiter dans le cadre restreint de cet article et je me limiterai ici à quatre propositions qui me semblent congruentes par rapport à l'expérience des projets dont il a été question dans cet article.

- Parce que des concepts tels que «environnement » et «enjeu environnemental » sont socialement construits, les curriculums d'éducation relative à l'environnement doivent engager les professeurs et les étudiants dans l'étude des modalités de la construction sociale de ces concepts au sein de certains contextes et de certaines communautés. Des formes appropriées de recherche en éducation relative à l'environnement doivent également reconnaître et comprendre le rôle et la nature de l'évaluation et de la critique sociale, à la fois dans les curriculums éducatifs et dans la recherche en éducation.

- Parce que des concepts tels que « environnement » et "enjeu environne- mental » sont socialement construits de diverses manières dans différents contextes environnementaux, sociaux, culturels, politiques et historiques, des formes appropriées de recherche doivent être sensibles aux caractéristiques de communautés spécifiques (les approches de recherche dont la rigueur repose sur des designs fixes et prédéfinis sont donc moins appropriées). Il s'agit là d'une observation plus spécifique faite il y a environ 20 ans au sujet de l'innovation en matière d'évaluation :

Compte tenu de la complexité des contextes d'innovation en évaluation, il est souvent inapproprié d'utiliser des approches d'évaluation se basant uniquement sur une efficacité du programme définie selon des normes ou des critères prédéterminés. De telles approches évaluatives tendent à être insensibles autant en ce qui a trait à l'évolution de l'innovation au cours du processus qu'aux contributions qualitatives et plus subjectives que les participants ont à offrir dans le cadre de l'innovation en évaluation. (Kemmis et Robottom, 1981, traduction libre)

- Affirmer que des concepts tels que «environnement » et « enjeu environnemental » sont socialement construits revient aussi à dire que leur construction particulière est fonction de valeurs ainsi que d'intérêts humains à la fois subjectifs et particuliers. Des formes appropriées de recherche doivent être capables de traiter des valeurs et des intérêts humains qui ont historiquement formé, contraint et défini les enjeux environnementaux (comme constructions sociales particulières) plutôt que de les nier et de les marginaliser. Il est crucial de porter un intérêt aux catégories interprétatives et à la signification des réalités pour les personnes concernées.

- Parce que la manière selon laquelle des concepts tels que «environnement " et «enjeu environnemental » sont socialement construits est influencée par des valeurs et des intérêts particuliers et afin que la recherche soit productive et utile dans la pratique (qu'elle "génère de nouveaux savoirs et les rende publics "), la recherche en éducation relative à l'environnement doit impliquer des réseaux de participants dans un processus d'explicitation et d'évaluation critique des modalités selon lesquelles des constructions 
spécifiques d'enjeux environnementaux viennent à se former et à être définies par certains intérêts humains particuliers.

\section{Conclusion}

41 internationaux centrés sur la communauté. L'un d'eux traitait des processus participatifs pour l'élaboration d'un programme d'éducation relative à l'environnement dans un contexte marqué par des changements sociaux et politiques majeurs. L'autre projet traitait des processus participatifs au sein de communautés cherchant à concevoir et à mettre en œuvre une nouvelle vision pour leur environnement local.

Ces deux exemples servent à illustrer la nature des enjeux d'environnement et de développement ainsi que les processus complexes et souvent politiques par lesquels de telles problématiques sont résolues. Il apparaît clairement que les enjeux environnementaux sont complexes, contextuels et socialement construits.

Il est ici avancé que la nature fondamentale de construction sociale qui caractérise l'« environnement », la « culture » et le « développement » a des incidences sur le type de recherche que nous adoptons pour explorer ces réalités dans le domaine de l'éducation relative à l'environnement. Plus précisément, il est suggéré que la recherche en éducation relative à l'environnement implique des réseaux de participants dans le processus d'explicitation et d'évaluation critique des manières selon lesquelles les enjeux environnementaux sont socialement construits, à la confluence d'intérêts humains spécifiques et divergents.

\section{BIBLIOGRAPHIE}

Alblas, A.H., Van den Bor, W. et Wals, A.E.J. (1994). Learning for responsibility and care : Integrating environmental issues in agricultural education. Wageningen, The Netherlands : Wageningen Agricultural University, Department of Agricultural Education.

Aleixandre, M. et Gayoso, I. (1996). An approach to introducing environmental education into the science methods course in teacher education. Environmental Education Research, 2 (1), 27-39.

Barkin, D. (2000). Overcoming the neoliberal paradigm : Sustaining popular development.

Journal of Developing Societies, 16(1), 163-180.

Boschhuizen, R. et Brinkman, F.G. (1995). The concept of cycles for environmental education. Environmental Education Research, 1(2), 147-158.

CADISPA. (2001). C.A.D.I.S.P.A. Glasgow : University of Strathclyde.

Diduck, A. (1999). Critical education in resource and environmental management : Learning and empowerment for a sustainable future. Journal of Environmental Management, 75, 85-97. 
Dove, J. (1996). Student teacher understanding of the greenhouse effect, ozone layer depletion and acid rain. Environmental Education Research, 2(1), 89-100.

Fien, J. (1997). Stand up, stand up and be counted : Undermining myths of environmental education. Australian Journal of Environmental Education, 13, 21-26.

Hampson, I. et Fagan, G. (1997). Local people, partnerships and rural sustainable development. Glasgow : CADISPA, Faculty of Education, University of Strathclyde.

Harris, J. et Robottom, I. (1997). Postgraduate environmental education research : Meeting the needs of the community. Australian Journal of Environmental Education, 13, 49-54.

House, E., Eide, K. et Kelly-Laine, K. (1994). In-depth study of environmental education policy

- Austria (Working Document). Braunschweig, Germany : OECD Review Team.

Izadi, P. et Kurtakko, K. (1994). Dynamics and management of change; possibilities of integrative consultation (Research Report). Rovaniemi : OECD/CERI Project, Environment and School Initiatives.

Kemmis, S. et Robottom, I. (1981). Principles of procedure in curriculum evaluation. Journal of Curriculum Studies, 13(2), 151-155.

LeGrange, L., Makou, T., Neluvhalani, E., Reddy, C. et Robottom, I. (1999). Professional selfdevelopment in environmental education : the case of the 'Educating for Socio-Ecological Change Project'. Unpublished manuscript, Grahamstown, South Africa.

Lotz, H. et Robottom, I. (1998). Environment as text : Initial insights into some implications for professional development in environmental education. Southern African Journal of Environmental Education, 18, 19-28.

Mrazek, R. (dir..). (1993). Alternative paradigms in environmental education research. Troy, Ohio : North American Association for Environmental Education.

Reddy, C. (2002). Restoring links. Communication personnelle, non publiée.

Robottom, I. (2000). Environmental education and the issue of coherence. Themes in Education, 1(3), 227-241.

Robottom, I. (1996). Permanently peripheral ? Opportunities and constraints in Australian environmental education. Southern African Journal of Environmental Education, 16, 44-56. Robottom, I. (1987). Contestation and consensus in environmental education. Curriculum

Perspectives, 7(1), 23-27.

Robottom, I. et Kyburz-Graber, R. (2000). Recent international developments in professional development in environmental education : Reflections and issues. Canadian Journal of Environmental Education, 5, 1-19.

Robottom, I. et Hart, P. (1995). Behaviorist EE research : Environmentalism as individualism. Journal of Environmental Education, 26 (2), 5-9.

\section{RÉSUMÉS}

En se basant sur deux récents projets environnementaux internationaux centrés sur la communauté, cet article explore les enjeux relatifs à la nature, au statut et au rôle de la recherche en éducation relative à l'environnement. À la lumière de ces expériences, au sein de 
deux cultures différentes, certaines caractéristiques des projets de développement à caractère environnemental centrés sur la communauté sont décrites, en mettant en relief la complexité et le caractère contextuel des processus de résolution de problèmes environnementaux. L'article aborde aussi la question des implications de telles caractéristiques pour une recherche visant à reconnaître et respecter les relations au sein de contextes communautaires.

This article will draw upon two recent international community-based environmental projects to explore issues concerning the nature, status and role of research in environmental education. A number of features of community-based environment development projects in two different cultures will be described, illustrating the complexity and contextuality of the processes by which environmental issues are resolved. The implications for research that seeks to acknowledge and respect relationships within community contexts will also be considered.

\section{INDEX}

Index géographique : Afrique du Sud, Australie, Écosse

Keywords : community, research methodologies, environmental education. teachers training, case study, community development

Mots-clés : communauté, méthodologies de recherche, éducation relative à l'environnement, formation des enseignants, étude de cas, développement communautaire

\section{AUTEURS}

\section{IAN ROBOTTOM}

Professeur associé à la Faculté d'éducation de l'Université de Deakin en Australie, il y est également coordonnateur du programme de maîtrise en éducation. Ses intérêts de recherche en éducation relative à l'environnement et en enseignement des sciences ont trait entre autres au développement curriculaire, au développement professionnel et à la recherche participative centrée sur la communauté. Ian Robottom a largement contribué à enrichir la réflexion sur la recherche en éducation relative à l'environnement, particulièrement en ce qui concerne le courant critique. 